\title{
Teaching optical fiber communications and optical data storage techniques using a compact disc player
}

Pierre Lane, Ronnie Van Dommelen, Michael Cada

Pierre Mark Lane, Ronnie F. Van Dommelen, Michael Cada, "Teaching optical fiber communications and optical data storage techniques using a compact disc player," Proc. SPIE 3190, Fifth International Topical Meeting on Education and Training in Optics, (8 December 1997); doi: $10.1117 / 12.294409$ 1997, Delft, Netherlands 


\title{
Teaching optical fiber communications and optical data storage techniques using a compact disc player
}

\author{
Pierre Lane, Ronnie Van Dommelen, Michael Cada \\ DalTech $^{\mathrm{a}}$, Dalhousie University, Department of Electrical Engineering \\ P.O. Box 1000, Halifax, NS B3J 2X4, Canada
}

\begin{abstract}
The principles of optical fiber communication and optical data storage have evolved over the past decade into industry standards for transmission, distribution, storage, and archival of digital audio, multimedia, and computer data. The fundamentals of these sophisticated systems are traditionally taught in the lecture hall, and laboratory exposure to the hardware is at the device rather than the system level.

In our educational approach, after the student has been introduced to the device basics, the compact disc audio player is used as a tool to teach the fundamentals of optical communication and storage. The ubiquitous compact disc audio system, from analog input, through optical storage and distribution, to audio reproduction, provides an excellent model of a complete real world optical transmission and storage system.

The laboratory time is divided into three segments: Introduction to Electro-Optic Devices, Optical Storage, and Optical Communication. During the introduction, students learn the basics of emitters, detectors, and optical fiber. The optical data storage and retrieval function of the compact disc player is investigated in the second segment. Students are introduced to optical information storage techniques, information density limits, the optical pick-up, laser diodes, photodiode arrays, eye patterns, and the tracking and focussing sub-systems. In the third segment, the compact disc system is used as a model of an entire optical communication system. Students are introduced to sampling and quantization, channel encoding techniques, modulation, high-speed transmitters and receivers, demodulation, decoding, error correction, digital signal processing, and digital-to-analog conversion.
\end{abstract}

Keywords: Education in Optics, Compact disc, Optical communication, Optical data storage

\section{INTRODUCTION}

The household compact disc (CD) player represents an absolute marvel of modern engineering. This amazing consumer product is realized through the marriage of several sophisticated systems and technologies. Each system illustrates fundamental principles from a variety of engineering disciplines including optics, communication theory, digital signal processing, and control engineering. The $\mathrm{CD}$ therefore provides an excellent example of the application of basic physics principles ${ }^{1}$. The household $\mathrm{CD}$ player is an excellent teaching tool because it exemplifies a great deal of technology and at the same time it is very cost effective.

Optical data storage techniques have emerged as the preferred choice for the distribution and storage of digital audio, multimedia, and computer data. The data density ${ }^{2}$ of an audio $\mathrm{CD}$ is approximately 100 megabit $\mathrm{cm}^{-2}$. The new digital

${ }^{2}$ DalTech, formally known as the Technical University of Nova Scotia (TUNS), was amalgamated with

Dalhousie University, and became a college within the framework of Dalhousie, on April 1, 1997. 
versatile disc (DVD) format, which was being released at the time of writing, has a data density greater than 500 megabit $\mathrm{cm}^{-2}$, and consequently boasts almost 100 times the data density of a typical magnetic removable-media drive $\left(6\right.$ megabit $\left.\mathrm{cm}^{-2}\right)$. DVDs with a total capacity of 17 gigabytes (four information layers) will be available soon.

The CD system was designed on the basis of communications concepts ${ }^{3}$. It can be considered as a transmission system that brings sound from the studio into the living room ${ }^{4}$. This makes the compact disc player an ideal tool for teaching the principles of modern digital communication. Students gain laboratory exposure to sophisticated modulation and coding schemes that are usually only discussed in the lecture hall.

This paper discusses how the CD player is incorporated into the laboratory sessions of the Optical Electronics course at DalTech, Dalhousie University. The laboratory time is divided into three segments: Introduction to Electro-Optic Devices, Optical Storage, and Optical Communication.

\section{ELECTRO-OPTIC DEVICES}

Students are introduced to basic electro-optics in a series of short classical laboratory experiments. These experiments are performed before the $\mathrm{CD}$ players are introduced in order that students get an appreciation for the electro-optic components. Experiments are designed to teach students about reflection, transmission, polarization, interference, diffraction, photoresistors, diodes, and transistors, LEDs and their radiation patterns, optocouplers, optical fibers, analog and digital modulation schemes, and power and rise time budgets.

\section{OPTICAL STORAGE}

Optical data storage techniques are investigated using the $\mathrm{CD}$ player. Students attend a short seminar where they are introduced to optical storage techniques, information density limits, the optical pick-up, laser diodes, photodiode arrays, eye patterns, modulation, coding, and the tracking and focussing sub-systems. The information discussed in the seminar is available from numerous sources ${ }^{1,35-8}$. A series of six experiments, designed to convey these ideas, follow over the next two laboratory sessions. Groups of students work at stations equipped with a CD player that has been setup for a particular experiment. There are equal numbers of stations and groups so that students can cycle through the experiments.

\subsection{Optical pick-up}

The optical pick-up's objective lens, beamsplitter, photodiode array, laser diode, and diffraction grating are identified and examined by the students. The photodiode array is removed from the pick-up and examined under a microscope. An optical microscope image of the photodiode array from the CD player used at DalTech (Emerson model CD 230) is shown in Figure 1. The quadrant detector and tracking photodiodes are clearly visible and the scale is 125 times. Students determine the slit spacing of the diffraction grating by observing its diffraction pattern under HeNe laser light illumination.

\subsection{Optical information density}

Students learn that optical information density is limited by diffraction. The Information density is determined primarily by the optical pick-up and is proportional to the NA of the objective lens squared and inversely proportional to the illuminating wavelength squared ${ }^{4}$. Students determine the track pitch of a CD by illuminating it with laser light and observing its diffraction pattern ${ }^{9-11}$. The track pitch is used to estimate the length of the spiral track ${ }^{1}$ on the $C D$, its total channel information capacity in bits ( 3 bits can be coded using $0.9 \mu \mathrm{m}$ of spiral track ${ }^{6}$ ), and the total duration of music that can be recorded (192 bits of PCM stereo audio per frame of 588 channel bits ${ }^{4}$ ). 


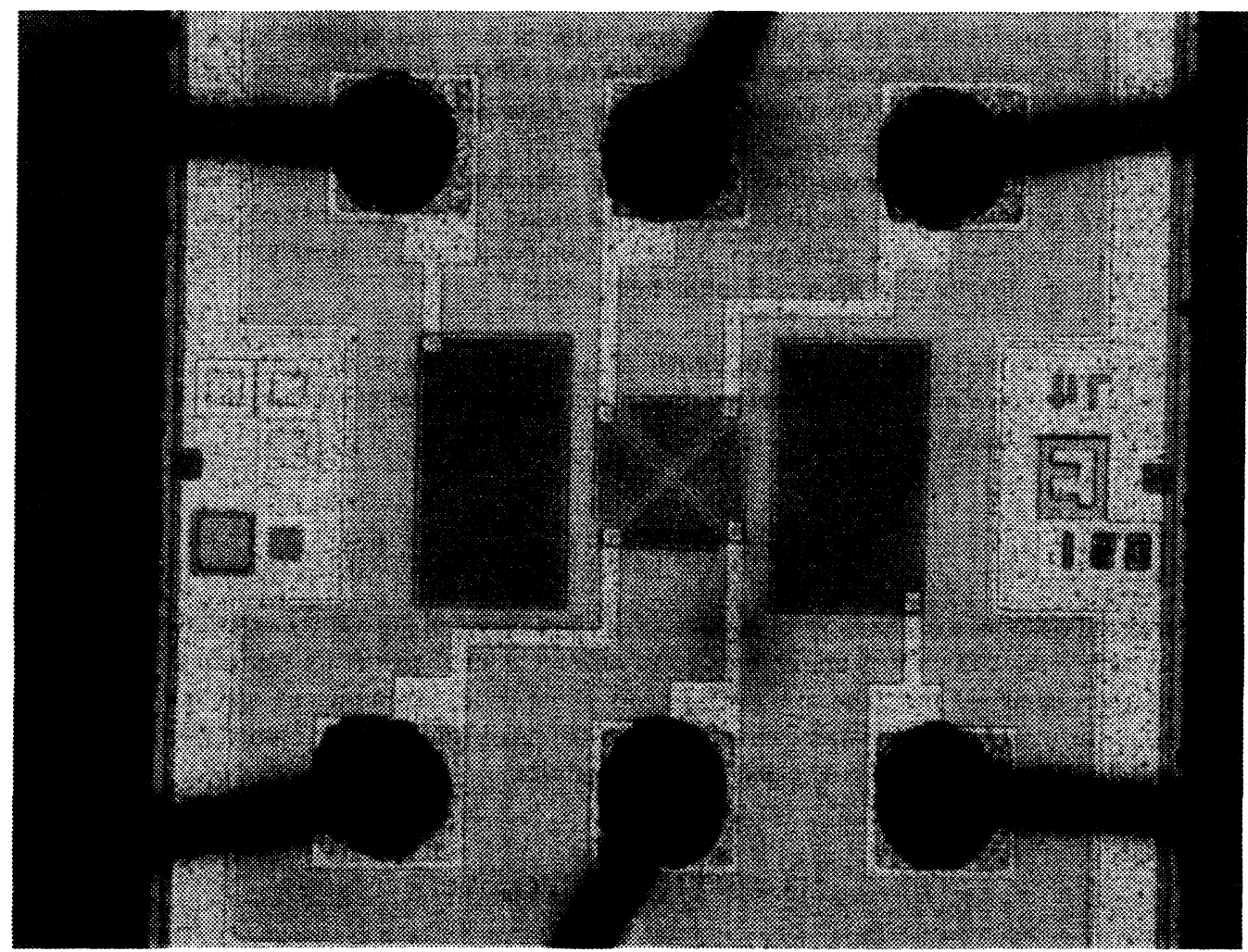

Figure 1 Optical microscope image of the photodiode array from the CD player used in the undergraduate laboratory at DalTech. The quadrant detector and tracking photo diodes are clearly visible. The magnification is 125 times.

\subsection{Angular and linear velocity}

Students determine that the $\mathrm{CD}$ audio system uses constant linear velocity for optical read-out and recording. The angular velocity of the disc is measured at five to ten equally spaced intervals over the entire length of the $C D$. The angular velocity measurements are plotted versus $1 / r$. A linear fit indicates the $C D$ system uses constant linear velocity (CLV) during readout. The disc radii $r$ can be determined from the elapsed time displayed by the CD player when the velocity measurements were made. The disc's angular velocity is proportional to the pulse width (or some other signal parameter) of the signal driving the spindle motor. Other optical information techniques, such as analog video discs ${ }^{5}$, use constant angular velocity (CAV).

\subsection{The eye pattern}

The eye pattern can reveal useful information about the noise margin, timing sensitivity, and expected performance of a communication system ${ }^{12}$. It consists of a superposition of all possible symbol to symbol transitions. A good quality communication system is characterized by a fully open eye while a partially closed eye indicates inter-symbol interference. Students are typically exposed to eye patterns generated by systems driven with pseudo-random number generators. The CD player provides an excellent source of a real-world eye pattern. A partially closed eye can be demonstrated by increasing the resistive load on the quadrant detector. The point at which inter-symbol interference begins is observable in the eye pattern and immediately obvious by the degradation in audio quality.

\subsection{Constant linear velocity}

Students determine the CLV of a rotating disc and compare their value to the CD specification. An oscilloscope is used to measure the duration of the shortest run length in the eight-to-fourteen (EFM) signal eye pattern. The shortest run 
length corresponds to a 3-pit pattern ${ }^{6}$ and is represented on the CD by a pit or land of length $0.9 \mu \mathrm{m}$. The disc's CLV is simply its minimum run length divided by its eye pattern duration.

\subsection{CIRC error correction}

Students evaluate the performance of the CD's error detection and correction system during the last optical storage experiment. The CD system uses a Cross-Interleaved Reed-Solomon Code (CIRC) for detection and correction ${ }^{13}$. It is possible to simulate burst errors, such as scratches, finger prints, and dropouts, by chopping the EFM signal between the transimpedance amplifier and the quantizer. The consequences of chopping the signal to simulate scratches are observed by monitoring the status of a CIRC error flag and listing to the quality of the audio.

The Yamaha chipset used in the CD players at DalTech was designed with a CIRC error correction flag. This flag was included on the chip by the manufacturer for diagnostic purposes and the CD player itself does not make use of it. The error correction flag may be polled at regular intervals to determine if the last frame was decoded correctly or if error correction was required. External circuitry was designed and realized to count the number of 24 byte frames that were correctly decoded by each of the two CIRC error correcting stages. In addition, the external circuitry counts the number of frames decoded by each stage where a single correction was required, where a double correction was required, and where correction of that frame was impossible. The CIRC status counts are periodically uploaded to a PC which provides a real-time graphical representation of the frame error rate, the single-correction rate, and the double-correction rate, for each of the two CIRC stages.

The frame error rate should be very close to zero when the information signal is not chopped. The maximum completely correctable burst error length ${ }^{13}$ can be estimated by chopping the information signal once every disc rotation and increasing the duty cycle of the chop until the errors in the signal can no longer be corrected. The duration of a single chop multiplied by the disc CLV corresponds to the maximum completely correctable burst error length. The two stage CIRC design specifies a maximum completely correctable burst error length of about 4000 data bits, corresponding to a track length of $2.5 \mathrm{~mm}$.

\section{OPTICAL FIBER COMMUNICATION}

The compact disc system is used in the third segment as a model of an entire optical communication system. Students are introduced to sampling and quantization, channel encoding techniques, modulation, high-speed transmitters and receivers, demodulation, decoding, error correction, digital signal processing, and digital-to-analog conversion.

\subsection{Sampling, channel encoding, and modulation}

The original analog audio signal is sampled at $44.1 \mathrm{kHz}$ and uniformly quantized with 16 bits of resolution for each of the left and right channels ${ }^{6}$. Eight parity bytes are added to each frame of 24 audio bytes using two CIRC stages to facilitate error detection and/or correction at the receiver. The coded audio data is modulated with eight-to-fourteen modulation (EFM) to produce the $4.32 \mathrm{Mb} / \mathrm{sec}$ channel bit stream ${ }^{4}$.

The audio CD therefore represents a convenient source of a coded and modulated signal for either observation on an oscilloscope or transmission over an optical fiber. Producing a similar data stream with a signal generator and subsequent signal processing would require several pieces of expensive test equipment which are usually not available in an undergraduate teaching laboratory. The complex signal can easily be recovered and extracted from an inexpensive CD player. The students can supply the CDs to suit their own musical tastes.

\subsection{Optical fiber communication link}

A communication link is designed and constructed by groups of students that will transmit the modulated 4.32 megabit per second signal, extracted from their CD player, over a length of optical fiber at a predetermined bit or frame error rate. A block diagram of the optical communication link is shown in Figure 2. Students are expected to design and build 

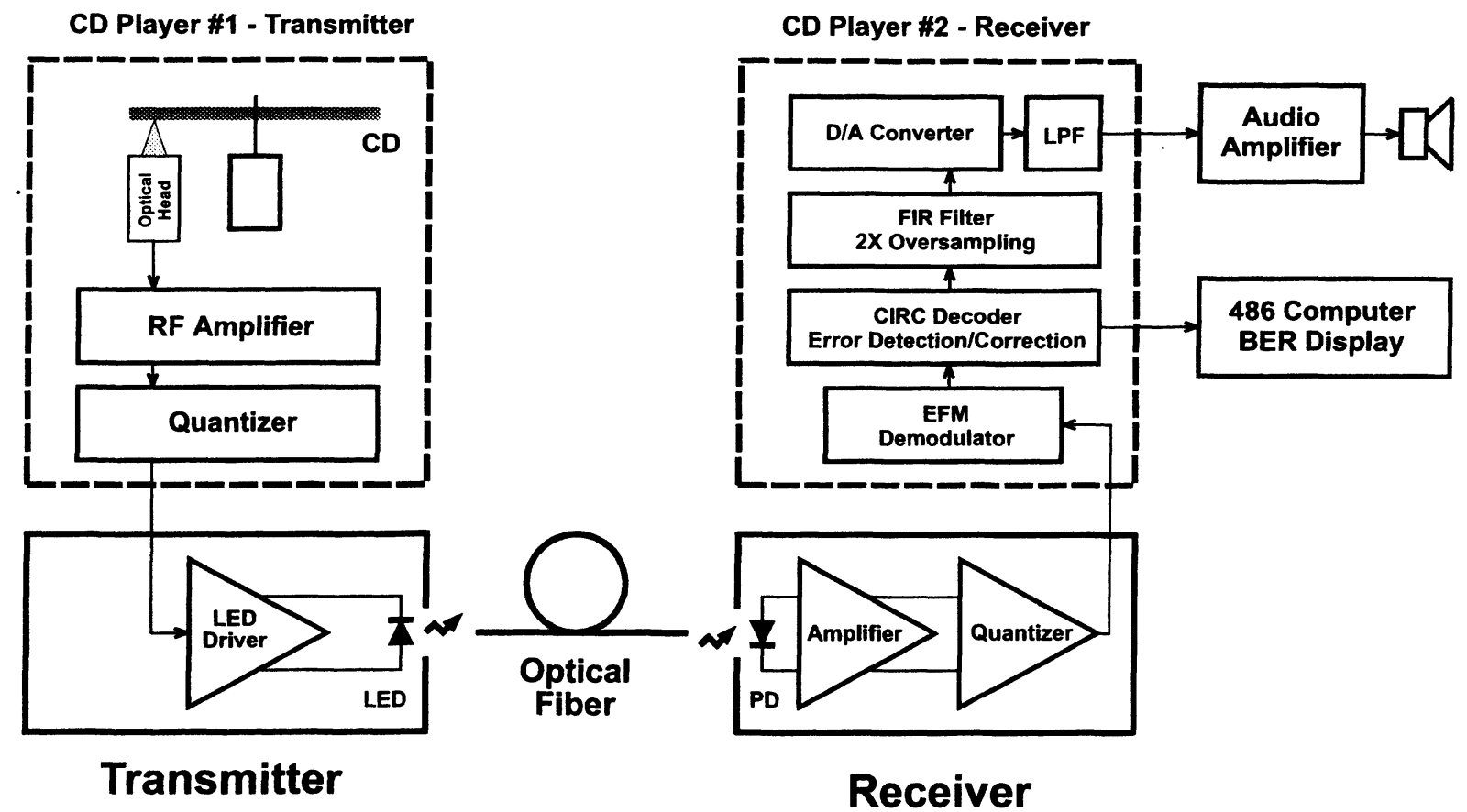

Figure 2 Block diagram of the $\mathrm{CD}$ optical communications link. The channel bit stream from $\mathrm{CD}$ player \#1 is transmitted across an optical fiber to CD player \#2 where it is demodulated, errors are detected and corrected (if possible), and the original analog audio signal is reconstructed. The channel bit rate is 4.32 megabits per second.

the transmitter and receiver circuits. The coded and modulated digital audio signal is extracted from a CD player and made available to the student through a BNC connector. The transmitter and receiver pair is designed to transmit the signal over $60 \mathrm{~m}$ of plastic fiber. The received signal is fed into to a second $C D$ player which demodulates the signal, performs error detection and/or correction, and reconstructs the original analog audio signal. The bit error rate (actually the frame error rate) of the communication link can be monitored using the error correction flag as was described previously in section 3.6 .

Students are expected to apply the theory and design procedures discussed in the lectures to the design and construction of their optical communication system. As part of the design, students are required to prepare rise time and power budgets. The design rise time and power budgets can be compared with the realized bandwidth and measured optical powers after the link has been characterized. A list of suggested components available in the laboratory is made available to the students. A good design and the proper choice of components will produce an optical data link that will transmit the CD signal across $60 \mathrm{~m}$ of plastic fiber at a very acceptable error rate. The performance of the optical link can be assessed qualitatively by examining its eye diagram and quantitatively by noting the frame error rate. The optical link can be characterized by the students using a number of different fiber lengths. For example, the frame error rate using a $60 \mathrm{~m}$ cable can be compared to that of the same transmitter and receiver with a $30 \mathrm{~m}$ cable.

\subsection{Demodulation, error correction, and digital signal processing}

The signal processing electronics required to demodulate, detect and (if possible) correct channel errors, and then oversample, are all integrated on one LSI chip in the CD player used at DalTech. Access to the internal signals is limited to the channel bit stream input, the oversampled digital audio output, and the CIRC status flag. These signals may be observed, either on an oscilloscope or acquired by a computer through a digital $\mathrm{V} / \mathrm{O}$ board, and can provide numerous insights into the digital post-processing of the received signal. It is particularly useful to be able to listen to the quality of the audio that is transmitted across the fiber and relate this to the quantitative frame error rate and the qualitative eye diagram. 


\section{CONCLUSIONS}

The compact disc player is a synergy of optics, communication theory, digital signal processing, and control engineering. It provides an excellent example of how basic physical principles can be applied to complex tasks such as optical data storage and optical fiber communication. Because the CD system was designed on the basis of communications concepts, it provides an excellent and cost effective teaching model.

\section{ACKNOWLEDGMENTS}

The authors would like to acknowledge support from the technical staff at DalTech, in particular, Christopher Hill and Mark LeBlanc, for their technical contributions and software development.

\section{REFERENCES}

1. J.A. Cope, "The physics of the compact disc," Phys. Educ., Vol. 28, pp. 15-21, 1993.

2. A.E. Bell, "Next-Generation Compact Discs," Scientific American, pp. 42-46, July 1996.

3. J.B.H. Peek, "Communications Aspects of the Compact Disc Digital Audio System," IEEE Communications Magazine, Vol. 23, pp. 7-15, February 1985.

4. J.P.J. Heemskerk and K.A. Schouhamer Immink, "Compact Disc: system aspects and modulation," Philips Tech. Rev. (Special Issue), Vol. 40(6), pp. 157-164, 1982.

5. G. Thomas and W. Ophey, "Optical Recording," Physics World, Vol. 3, pp. 36-41, December 1990.

6. M.G. Carasso, J.B.H Peek, and J.P. Sinjou, "The compact disc digital audio system," Philips Tech. Rev. (Special Issue), Vol. 40(6), pp. 151-156, 1982.

7. T.D. Rossing, "The Compact Disc Digital Audio System," Phys. Teach., Vol. 25, pp. 556-562, 1987.

8. K.C. Pohlmann, Principles of Digital Audio, Second Ed., Chapter 12, Sams, Carmel, 1989.

9. C. Nöldeke, "Compact Disc Diffraction," Phys. Teach., Vol. 28 , pp. 484-485, 1990.

10. J.E. Kettler, "The compact disc as a diffraction grating," Am. J. Phys., Vol 59(4), pp. 367-368, 1991.

11. H. Kruglak, "The compact disc as a diffraction grating," Phys. Educ., Vol. 25, pp. 255-256, 1990.

12. A.B. Carlson, Communication Systems. An Introduction to Signals and Noise in Electrical Communication, Third Ed., pp. 386-387, McGraw Hill, New York, 1986.

13. H. Hoeve, J. Timmermans, and L.B. Vries, "Error correction and concealment in the compact disc system," Philips Tech. Rev. (Special Issue), Vol. 40(6), pp. 166-172, 1982. 